\title{
Mechanical Characterization of Partially Crystallized Sphere Packings
}

\author{
M. Hanifpour, ${ }^{1}$ N. Francois, ${ }^{2, *}$ S. M. Vaez Allaei, ${ }^{1}$ T. Senden, ${ }^{2}$ and M. Saadatfar ${ }^{2, *}$ \\ ${ }^{1}$ Department of Physics, University of Tehran, Tehran 14395-547, Iran \\ ${ }^{2}$ Department of Applied Mathematics, Research School of Physics and Engineering, \\ Australian National University, Canberra, Australian Capital Territory 0200, Australia
}

(Received 22 January 2014; published 1 October 2014)

\begin{abstract}
We study grain-scale mechanical and geometrical features of partially crystallized packings of frictional spheres, produced experimentally by a vibrational protocol. By combining x-ray computed tomography, $3 \mathrm{D}$ image analysis, and discrete element method simulations, we have access to the 3D structure of internal forces. We investigate how the network of mechanical contacts and intergranular forces change when the packing structure evolves from amorphous to near perfect crystalline arrangements. We compare the behavior of the geometrical neighbors (quasicontracts) of a grain to the evolution of the mechanical contacts. The mechanical coordination number $Z_{m}$ is a key parameter characterizing the crystallization onset. The high fluctuation level of $Z_{m}$ and of the force distribution in highly crystallized packings reveals that a geometrically ordered structure still possesses a highly random mechanical backbone similar to that of amorphous packings.
\end{abstract}

DOI: 10.1103/PhysRevLett.113.148001

PACS numbers: 45.70.-n, 05.65.+b, 61.43.-j, 81.10.Aj

Noncohesive granular materials can show substantial rigidity under compression but may suffer global structural rearrangements when vibrated or sheared, a feature common to many soft materials and of crucial importance in designing industrial processes [1-4]. The mechanical stability of these systems is a rich nonlinear problem [5,6] which intrinsically relies on a complex interplay between geometrical and mechanical constraints at the "grain scale." The intricate structure of granular materials renders these geometrical and mechanical features difficult to describe and therefore to model $[7,8]$.

Monodisperse frictional sphere packings naturally form amorphous structures which might massively crystallize under vibrations or shearing $[9,10]$. Friction introduces a sharp nonlinearity in grain contact laws which challenges our understanding of mechanically stable structures $[1,6,11,12]$. Numerous experimental studies have reported that stable disordered packings can be generated at densities ranging from $\phi \approx 0.55$ to $\phi \approx 0.64$ [13-16]; some numerical studies suggest that this density range might be even wider [17-21]. To obtain packing densities beyond the upper limit of the amorphous range, strong vibrations have to be applied, and crystalline clusters inevitably form in highly monodisperse packings [13]. Although this emerging order is known to enforce a sharp geometrical transition on the packing structure $[9,22-24]$, the role of the grain's mechanical interactions in producing stable crystalline packings has so far been poorly addressed.

Conversely, disordered packings of frictionless spheres have been extensively studied for their marginal stability, or isostaticity [1,7]. The mechanical stability of such isostatic structures is governed by subtle relations that constrain the force and the pair distribution function $[1,5,20,25]$.
Interestingly, recent theoretical studies suggest that a phase diagram for frictional disordered packings can be built on two parameters, which characterize the geometrical and mechanical features at the grain scale [26]. This diagram describes each grain's neighborhood via (i) the geometrical coordination number $Z_{g}$, a geometric parameter which allows us to estimate the number of close neighbors surrounding a bead, and (ii) the mechanical contact number $Z_{m}$ which characterizes the mechanical backbone by quantifying the force bearing grain contacts. This approach opens up new perspectives on how the geometrical and mechanical features at the grain scale conspire to produce mechanically stable packings. It has recently revealed interesting aspects of the crystallization of numerically generated frictionless packings [27]. These advances have been made by considering idealistic configurations, and as such, they raise many nontrivial questions about their pertinence to realistic (i.e., polydisperse and frictional) packings $[1,9,28]$.

To date, there has been no three-dimensional (3D) mechanical characterization of partially to fully crystallized packings made of frictional quasimonodisperse spheres. As a consequence, the experimental relevance of the concept of $\left(Z_{g}, Z_{m}\right)$ in characterizing crystalline frictional structures or the evolution of intergranular forces during crystallization are still unknown.

In this Letter, we study such packings produced under gravity using a vibrational protocol. Our experiments harness $\mathrm{x}$-ray computed tomography, 3D image analysis, and numerical simulations to investigate the evolution of $Z_{g}, Z_{m}$, as well as intergranular forces, while crystallization takes place. $Z_{m}$ is shown to be a key parameter in describing the crystallization onset. Our analysis reveals 
that $Z_{m}$ and the intergranular forces remain highly fluctuating quantities even in almost perfectly crystalline packings. The force distribution function in geometrically ordered structures is shown to evolve substantially with the level of crystallinity while retaining some features similar to those observed in disordered structures $[25,29]$.

We use monosized acrylic beads (diameter $d=1 \mathrm{~mm}$, polydispersity $=0.025 \mathrm{~mm}$ ) which are packed into large cylindrical containers (diameter $=66 \mathrm{~mm}$ ). The beads are covered with graphite to reduce electrostatic repulsion between the grains. The coefficient of friction between the beads is $\mu=0.7 \pm 0.1$. Our experimental protocol is based on compaction by an intense fluidization of the packing $[9,30]$. A batch of approximately 200,000 beads is initially poured into a container, forming a random packing with a volume fraction $\phi$ ranging from $60 \%$ to $63 \%$. The container is then placed on a shaker allowing for both vertical and horizontal vibrations. The vibrations are sinusoidal, with a frequency set to $f=50 \mathrm{~Hz}$. The vertical component of the acceleration is set to $2.5 \mathrm{~g}$ and is 5 times larger than the horizontal one. The container is vibrated for 20 seconds. The resulting packings show substantial crystallization with a global volume fraction ranging from $68.5 \%$ to $70 \%$, which is well beyond Bernal's limit $\left(\phi_{\text {Bernal }} \approx 0.64\right)$. Since all the vibrating parameters are fixed, this finite density range reflects the complex formation history of our crystalline frictional packings.

Figure 1(a) shows a 3D rendering of such a partially crystallized structure. The bright regions correspond to locally disordered aggregates of beads; a disordered core and the boundaries between different crystal domains are thus highlighted. Both random and crystalline phases coexist in the packing [see Figs. 1(b) and 1(c)].

Helical x-ray computed tomography is utilized to image the internal 3D structure of packings with a spatial resolution of $30 \mu \mathrm{m}[9,15,31-33]$. Four packings have
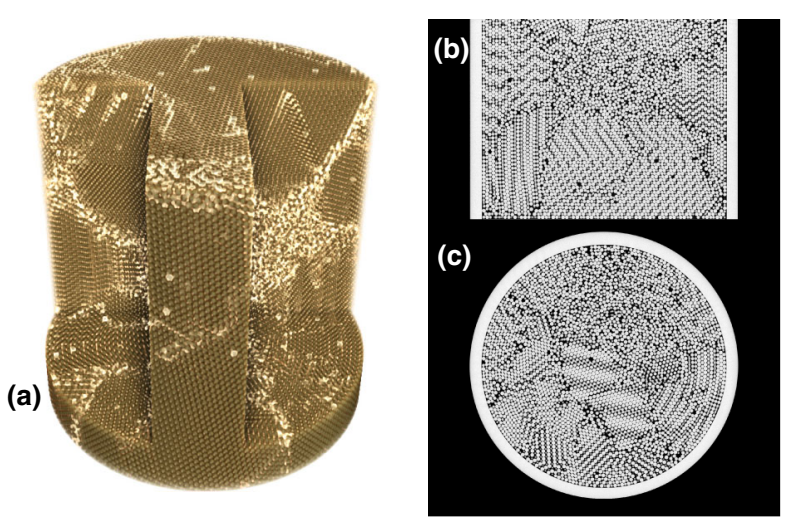

FIG. 1 (color online). (a) 3D visualization of a partially crystallized packing containing 200,000 beads. (b, c) 2D vertical and horizontal slices through the tomogram shown in (a). The cylindrical container is $66 \mathrm{~mm}$ in diameter and is filled with $1 \mathrm{~mm}$ diameter beads. been imaged: two jammed packings obtained by pouring and two partially crystallized packings. Our analyses have been carried out over nonoverlapping cubical subsets extracted from an inner region, four sphere diameters away from the container walls. Each subset contains 4000 beads. As a consequence of structural heterogeneity in partially crystallized packings, the subsets have a wide range of volume fractions ranging from $\phi=0.58$ to $\phi=0.73$.

To detect local structural changes in our packings, we employ an extension of the bond order parameter method [24,34] (see Ref. [35]). First, the third-order rotational invariant of rank six, $w_{6}$, is computed, and then we determine the half-height of its cumulative distribution, referred to as $w_{6}^{\text {hh }}$ (see inset of Fig. 2). This parameter has been shown to be an extremely sensitive measure of the onset of the local orientational order [24]. Figure 2 shows the variation of $w_{6}^{h h}$ with packing fraction $\phi$. There are two clear structural transitions: an abrupt increase at $\phi_{\text {Bernal }} \simeq$ 0.64 corresponding to the crystallization onset, and a plateau starting at $\phi_{c} \simeq 0.68$. Remarkably, these two densities identify clear transitions in the Voronoi volume fluctuations and the topology of packing polytetrahedral structures as observed in Ref. [9].

Each grain's bulk in the 3D digital tomogram is made up of a cluster of $\approx 19,000$ voxels. As a consequence, the precision in determining grain center and diameter is extremely high (around $10^{-3} \mu \mathrm{m}$ and $5.10^{-2} \mu \mathrm{m}$, respectively). It thus provides an accurate experimental measure of grain overlap, which in turn gives us access to the mechanical backbone. This direct approach allows us to determine the mechanical contacts for normal forces as low

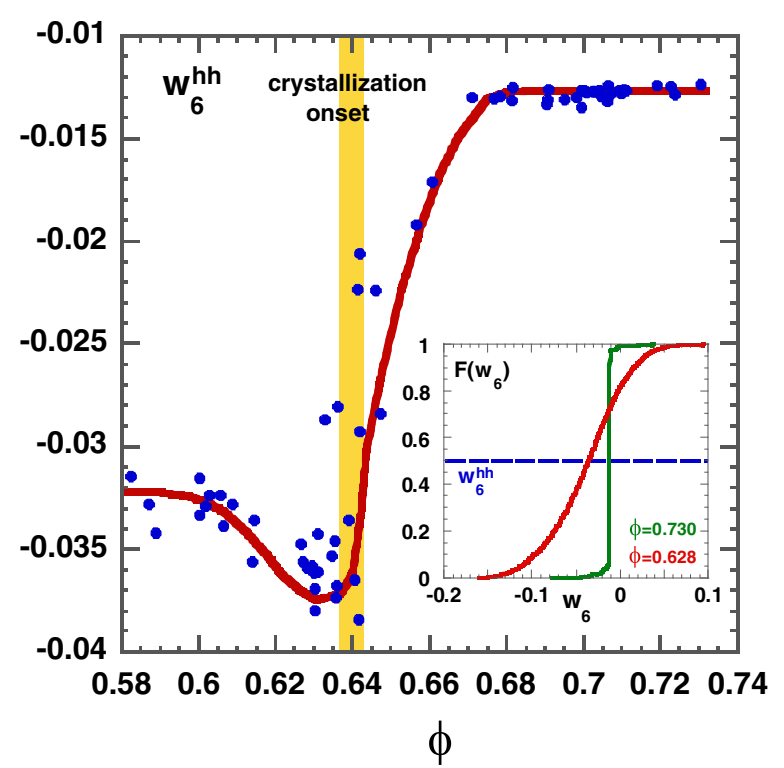

FIG. 2 (color online). Local order parameter $w_{6}^{\text {hh }}$ versus $\phi$. Inset: Calculation of $w_{6}^{\text {hh }}$ from the cumulative distribution $F\left(w_{6}\right)=\int_{-\infty}^{w_{6}} P(w) d w$ for two volume fractions $[P(w)$ is the PDF of $w_{6}$ ]. 

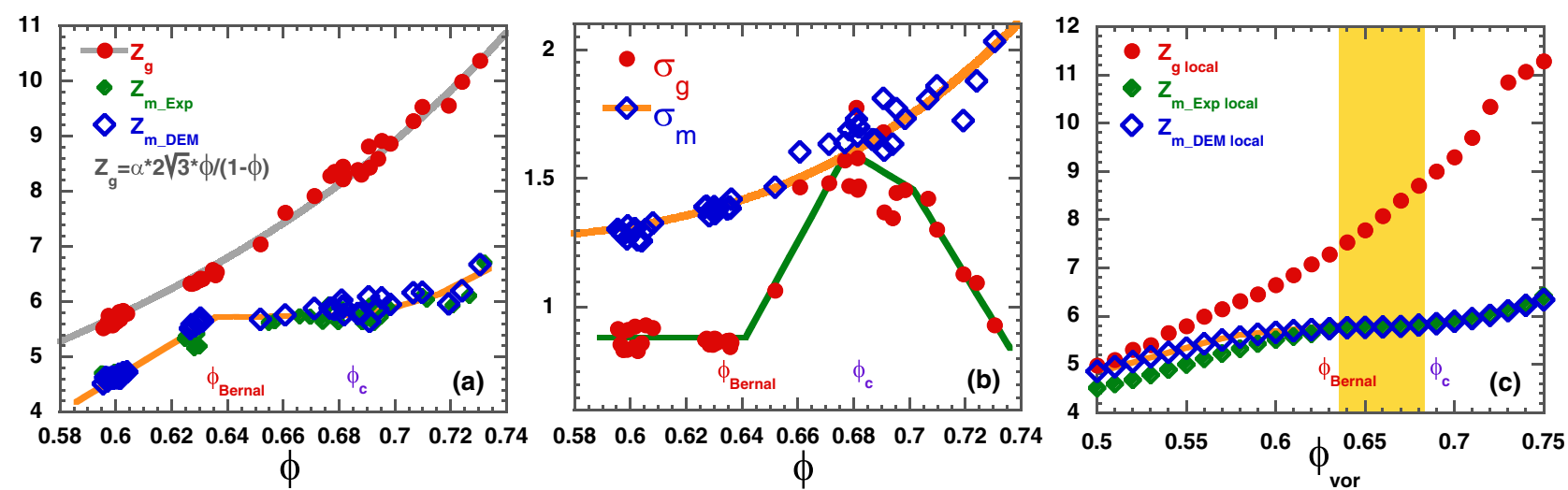

FIG. 3 (color online). (a) Geometrical $Z_{g}$ and mechanical $Z_{m}$ coordination numbers versus the volume fraction $\phi$. $Z_{m}$ is computed from both the direct estimation of grain overlaps (Exp) and the packing structure after DEM relaxation (DEM). $Z_{g}, Z_{m}$, and $\phi$ are averaged over 4000 bead subsets. $Z_{g}$ is fitted by $Z_{g}=\alpha \cdot 2 \sqrt{3} \phi /(1-\phi)$ [26] with $\alpha \approx 1.1$. (b) Standard deviation $\sigma_{g}$ and $\sigma_{m}$ of $Z_{g}$ and $Z_{m}$, respectively, versus $\phi$ computed over 4000 bead subsets. (c) $Z_{g}$ and $Z_{m}$ (Exp and DEM) averaged at the grain scale versus the local volume fraction $\phi_{\text {vor }}$.

as $10^{-4} \mathrm{~N}$, while the average normal force is $\left\langle F_{n}\right\rangle \approx$ $10^{-1} \mathrm{~N}$ in our experiments.

Force bearing contacts are an extremely sensitive feature of hard sphere packings [26], which are inevitably affected by intrinsic experimental limitations such as the finite spatial resolution or the binarization of the tomogram. To further assess the robustness of our mechanical characterization, experimental packing structures are subsequently post-processed by a discrete element method (DEM) code [36]. The DEM uses a Hertz-Mindlin contact model to compute the forces (both normal and frictional) between the grains (see Ref. [35]). During the DEM simulations, packing structures are relaxed under gravity. This second approach allows us to resolve the network of force bearing contacts with numerical precision. The results can be compared with the direct experimental characterization.

To explore how crystallization-induced grain rearrangements affect the mechanical and geometrical features of our packing at the grain scale, we investigate each grain's proximity in terms of its touching and almost-touching neighbors $[5,20,26]$.

We employ the approach developed in Refs. [26,27], which assumes that the topology of a grain neighborhood can be described by two different average coordination numbers: (i) the mechanical coordination number $Z_{m}$ which characterizes the contacts that bear forces, and (ii) the geometrical coordination number $Z_{g}$ which is defined as the maximum of the modified radial distribution function [26] (see Ref. [35]). $Z_{g}$ includes both forcebearing contacts and the number of grains close enough to be considered as immediate neighbors. It has recently been shown how these quasicontacts participate in the mechanical stability of marginally stable solids $[5,20]$.

Figure 3(a) shows that $Z_{g}$ and $Z_{m}$ evolve quite differently during the crystallization. $Z_{g}$ continuously increases over the entire range of volume fraction $\phi$. Its evolution can be fitted by $Z_{g} \approx 2 \sqrt{3} \phi /(1-\phi)$ [26].

Conversely, several regimes are observed in the evolution of $Z_{m}$ with $\phi$. In disordered packings $\left(\phi<\phi_{\text {Bernal }}\right), Z_{m}$ grows substantially with packing densification. As $\phi$ increases from 0.64 to 0.68 , Fig. 3(a) shows a plateau region in $Z_{m}$, where we measure $Z_{m} \approx 5.6$. Beyond $\phi_{c} \simeq 0.68, Z_{m}$ increases again up to $Z_{m} \approx 6.8$ at $\phi=0.73$. In highly crystalline packings, there is still a substantial difference between $Z_{g}$ and $Z_{m}$.

Thus, our experiments prove some level of universality of a mechanical feature recently observed in the numerical modeling of frictionless packings [27]: $Z_{m}$ stays constant for $\phi \in[0.64,0.68]$. Here we show that this feature is recovered in frictional packings, which were obtained by applying intense vibrations.

However, we stress three clear distinctions from the scenario presented in Ref. [27]: (i) $Z_{m}$ undergoes a sharp transition at the crystallization onset in packings for which $\mu$ is constant. (ii) For frictionless packings, $Z_{m}$ shows a plateau at $Z_{\text {plat }}=6$. In our experiments, we found $Z_{\text {plat }} \approx 5.6$; thus, $Z_{\text {plat }}$ might be friction dependent. (iii) Although the friction coefficient $\mu$ is constant, $Z_{m}$ increases with the compaction of disordered packings, in agreement with Ref. [36]. It highlights the importance of preparation history in the packing formation. It also suggests that a unique generalized isostatic condition relating $Z_{m}$ and $\mu$ does not characterize these frictional disordered packings which are thus hyperstatic [1]. In the case of perfectly monodisperse spheres, the presence of crystalline aggregates forbids the comparison between $Z_{m}$ and the classical isostatic counting [27]. However, the weak, yet finite, polydispersity of our spheres removes this constraint and ensures that our partially crystallized packings are hyperstatic [7].

At $\phi \geq 0.64$, there is an abrupt increase in the difference $\left(Z_{g}-Z_{m}\right)$ while $Z_{m}$ stays almost constant. It suggests that 
an increasing number of close neighbors (i.e., $Z_{g}-Z_{m}$ ) leads "touching" neighbors to roll or slide at the surface of each grain, until the grains reach a more crystalline configuration [37].

In Fig. 3(a), $Z_{m}$ and the packing fraction $\phi$ are averaged over 4000 grains. However, $Z_{m}$ can also be measured for individual grains and compared with the local volume fraction $\phi_{\text {vor }}=\left(\pi d^{3} / 6 V_{\text {vor }}\right)$, where $V_{\text {vor }}$ is the volume of the Voronoi cell surrounding a grain [9]. Figure 3(c) shows the trend of $Z_{m}$ versus $\phi_{\text {vor }}$, which consists of three distinct regions similar to those observed in Fig. 3(a). In particular, there is a clear plateau region in the range $\left[\phi_{\text {Bernal }}, \phi_{c}\right]$. We emphasize that this mechanical feature [Figs. 3(a) and 3(c)] is supported by direct experimental measurements based on the estimation of grain overlaps as well as the DEM results obtained with numerical precision.

Figure 3(b) shows the standard deviation $\sigma_{g}$ and $\sigma_{m}$ of the fluctuations in $Z_{g}$ and $Z_{m}$ versus $\phi$. A sudden rise in $\sigma_{g}$ signals the crystallization onset at $\phi_{\text {Bernal }}$, and these high levels of fluctuations persist for $\phi_{\text {Bernal }}<\phi<\phi_{c}$. Beyond $\phi_{c}, \sigma_{g}$ drops abruptly. Conversely, $\sigma_{m}$ increases steadily and peaks at $\sigma_{m} \approx 2$ for $\phi \approx 0.73$. Such evolution for $\sigma_{m}$ has been observed in simulations of monodisperse sphere packings [27]; thus, a high level of fluctuations in the mechanical backbone of almost crystalline structures may be independent of the low polydispersity of our spheres. Moreover, the persistence of a high fluctuation level $\sigma_{m}$ for $\phi>\phi_{c}$ along with a significant drop in the geometric fluctuations $\sigma_{g}$, reveals that a geometrically ordered structure can have a highly random mechanical backbone. A global picture emerges for highly crystallized packings, where the topological structure of the mechanical contact network might be mapped onto the sites of a regular lattice with random connectivity.

We now assess the changes in the intergranular forces that occur with the aforementioned evolution of the contact network topology. In Figs. 4(a) and 4(b), we show the probability density function (PDF) of the normal and tangential forces at the contact scale for increasing $\phi$. All PDFs have exponential tails which not only persist but also become more pronounced in highly crystalline structures. The enhancement of exponential tails is consistent with the presence of a mechanical contact network, which remains random and shows increasing fluctuations at high $\phi$ values. The 3D force PDFs evolve substantially with the level of crystallinity. This conclusion is in sharp contrast with the results obtained in a previous study [38] where the 2D distributions of normal forces measured at the boundaries of packings were found to be almost identical in amorphous and perfectly crystalline packings.

For $\phi>\phi_{\text {Bernal }}$, a pronounced peak grows at low forces $(F \approx 0.1\langle F\rangle)$ for both normal and tangential force components. In marginally stable, isostatic, disordered packings, the existence of weak forces is found to be a destabilizing
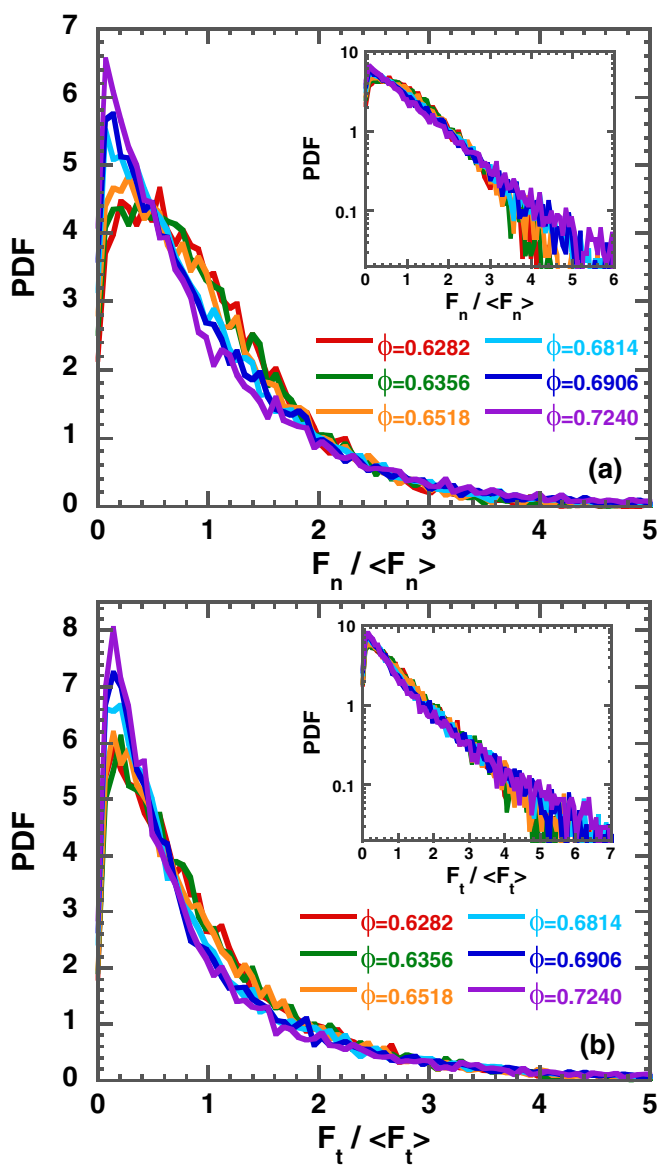

FIG. 4 (color online). PDFs of the normal and tangential intergranular forces, in (a) and (b), respectively, versus volume fraction $\phi$. The PDFs and the average force value $\langle F\rangle$ are computed over 4000 bead subsets. Insets: log-lin plot of the PDFs.

factor, while an abundance of quasicontacts plays a stabilizing role $[5,20]$. Interestingly, a crystal composed of weakly polydisperse frictionless beads can be isostatic and have properties similar to those of jammed matter [39]. Our frictional crystallized packings are hyperstatic and have a large number of quasicontacts, i.e., large $\left(Z_{q}-Z_{m}\right)$ values. Both features secure the mechanical stability of the packing and thus allow the presence of numerous weak forces within the mechanical backbone.

In summary, we have shown the existence of a plateau in the mechanical coordination number $Z_{m}$ in frictional packings with densities in the range $(0.64<\phi<0.68)$. This plateau might be a universal feature of partially crystallized packings for which there is, as yet, no theory. As the geometric order grows, so do the fluctuations in the force network. Consequently, an almost crystalline structure made of weakly polydisperse beads still possesses a highly random mechanical backbone. Moreover, the number of quasicontacts increases as crystallization takes place. We suggest that this behavior plays a role in the mechanical stability of partially crystallized packings and might influence the shape of the force PDF. Persistent exponential tails 
in the large force range and pronounced peaks in weak forces are observed in the force distributions beyond the crystallization onset, and they become even more pronounced with increasing $\phi$.

These novel experimental observations further support the conclusions drawn in recent numerical studies [39,40]. These results show that regular lattices made of weakly polydisperse beads [39] or highly ordered, yet imperfect, structures made of frictionless spheres [40] have mechanical properties best described by those of amorphous packings.

We thank E. Franklin for proofreading the final version and $\mathrm{M}$. Driol for her support. We thank the anonymous referees for their constructive reviews.

*nicolas.francois@anu.edu.au

†mos110@physics.anu.edu.au

[1] M. van Hecke, J. Phys. Condens. Matter 22, 033101 (2010).

[2] R. G. Larson, The Structure and Rheology of Complex Fluids (Oxford University Press, New York, 1999).

[3] F. Bolton and D. Weaire, Phys. Rev. Lett. 65, 3449 (1990).

[4] S. Hutzler, M. Saadatfar, A. van der Net, D. Weaire, and S. J. Cox, Colloids Surf. 323, 123 (2008).

[5] M. Wyart, Phys. Rev. Lett. 109, 125502 (2012).

[6] D. Bi, J. Zhang, B. Chakraborty, and R. P. Behringer, Nature (London) 480, 355 (2011).

[7] C. F. Moukarzel, Phys. Rev. Lett. 81, 1634 (1998).

[8] F. M. Schaller, M. Neudecker, M. Saadatfar, G. Delaney, G. E. Schröder-Turk, and M. Schröter, arXiv:1312.1327.

[9] N. Francois, M. Saadatfar, R. Cruikshank, and A. Sheppard, Phys. Rev. Lett. 111, 148001 (2013).

[10] K. E. Daniels and R. P. Behringer, Phys. Rev. Lett. 94, 168001 (2005).

[11] T. S. Majmudar and R. P. Behringer, Nature (London) 435, 1079 (2005).

[12] S. Papanikolaou, C. S. O'Hern, and M. D. Shattuck, Phys. Rev. Lett. 110, 198002 (2013).

[13] J. D. Bernal, Proc. R. Soc. A 280, 299 (1964).

[14] G. Y. Onoda and E. G. Liniger, Phys. Rev. Lett. 64, 2727 (1990).

[15] T. Aste, M. Saadatfar, and T. J. Senden, Phys. Rev. E 71, 061302 (2005).

[16] M. Jerkins, M. Schröter, H. L. Swinney, T. J. Senden, M. Saadatfar, and T. Aste, Phys. Rev. Lett. 101, 018301 (2008).

[17] S. Torquato, T. M. Truskett, and P. G. Debenedetti, Phys. Rev. Lett. 84, 2064 (2000).
[18] G. Parisi and F. Zamponi, Rev. Mod. Phys. 82, 789 (2010).

[19] P. Chaudhuri, L. Berthier, and S. Sastry, Phys. Rev. Lett. 104, 165701 (2010).

[20] P. Charbonneau, E. I. Corwin, G. Parisi, and F. Zamponi, Phys. Rev. Lett. 109, 205501 (2012).

[21] S. C. Kapfer, W. Mickel, K. Mecke, and G. E. SchröderTurk, Phys. Rev. E 85, 030301 (2012).

[22] A. V. Anikeenko and N. N. Medvedev, Phys. Rev. Lett. 98, 235504 (2007).

[23] A. V. Anikeenko, N. N. Medvedev, and T. Aste, Phys. Rev. E 77, 031101 (2008).

[24] B. A. Klumov, S. A. Khrapak, and G. E. Morfill, Phys. Rev. B 83, 184105 (2011).

[25] C. S. O’Hern, S. A. Langer, A. J. Liu, and S. R. Nagel, Phys. Rev. Lett. 86, 111 (2001).

[26] C. Song, P. Wang, and H. A. Makse, Nature (London) 453, 629 (2008).

[27] Y. Jin and H. A. Makse, Physica (Amsterdam) 389A, 5362 (2010).

[28] M. Saadatfar, A. P. Sheppard, T. J. Senden, and A. J. Kabla, J. Mech. Phys. Solids 60, 55 (2012).

[29] C. S. O'Hern, S. A. Langer, A. J. Liu, and S. R. Nagel, Phys. Rev. Lett. 88, 075507 (2002).

[30] P. Philippe and D. Bideau, Phys. Rev. Lett. 91, 104302 (2003).

[31] T. Aste, M. Saadatfar, and T. J. Senden, J. Stat. Mech. (2006) P07010.

[32] M. Saadatfar, N. Francois, A. Arad et al., SEG Las Vegas Annual Meeting, 2273-2281 (2012).

[33] T. Varslot, A. Kingston, G. Myers, and A. Sheppard, Med. Phys. 38, 5459 (2011).

[34] P. J. Steinhardt, D. R. Nelson, and M. Ronchetti, Phys. Rev. Lett. 47, 1297 (1981).

[35] See Supplemental Material at http://link.aps.org/ supplemental/10.1103/PhysRevLett.113.148001 for details regarding the experimental setup, the numerical simulation using DEM, the characterization of the geometrical coordination number $Z_{g}$ and the bond order parameter method.

[36] G. W. Delaney, T. D. Matteo, and T. Aste, J. Soft Matter 6, 2992 (2010).

[37] J. A. van Meel, D. Frenkel, and P. Charbonneau, Phys. Rev. E 79, 030201 (2009).

[38] D. L. Blair, N. W. Mueggenburg, A. H. Marshall, H. M Jaeger, and S. R. Nagel, Phys. Rev. E 63, 041304 (2001).

[39] R. Mari, F. Krzakala, and J. Kurchan, Phys. Rev. Lett. 103, 025701 (2009).

[40] C. P. Goodrich, A. J. Liu, and S. R. Nagel, Nat. Phys. 10, 578 (2014). 